\title{
Long-term Photometric and Spectral Study of Planetary Nebula Variability (1968-2005)
}

\author{
E. B. Kostyakova \\ Sternberg State Astronomical Institute of Moscow University, Moscow, Russia \\ email: ekost@sai.msu.su
}

\begin{abstract}
The results of long term observations of photometric $(U B V)$ and spectral variability of several PNe are presented. For the most variable nebulae, IC 4997 and NGC 6572, showing both photometric and spectral changes in time, the main physical parameters $\left(T_{e}, N_{e}\right.$ and $\left.T_{*}\right)$ and their time variations were estimated and discussed.
\end{abstract}

Keywords. planetary nebulae, photometry, spectroscopic observations, variability

A systematic study of planetary nebula variability was started in 1968 at the Crimean Station of Moscow University. During long-term examination we revealed several planetaries with well-marked variations of the integral $U B V$-brightness, viz. NGC 6572 , IC 4997, NGC 6891, Hu 2-1, and NGC 6543. The results of photoelectric observations (1968-1996) of seven nebulae were published in three catalogues (Kostyakova (1990); Kostyakova (1991); Arkhipova, Kostyakova \& Noskova (1994)) and by Kostyakova (1997). The method and analysis were described by Kostyakova, Arkhipova \& Savel'eva (1973).

Our long-term spectral study (1972-2005) of the same objects showed also noticeable changes in their spectra. The integral fluxes in the brightest nebular emission lines and in continua of central stars were measured on objective prism spectrograms obtained with a 50 -cm Maksutov meniscus telescope $(D / f=1: 4)$. The examined spectral region was $\sim \lambda 3400-5100 \AA$. In our spectral study, as in the photoelectrical one, we tried to keep a common procedure of observations, technique and reduction of material in order to obtain an extended, homogeneous series. We cannot reveal short-period variations of energy fluxes; our individual published results are usually averaged through every observational moonless season.

The most remarkable photometric and spectral behaviour was found for the well-known extremely young planetary nebula IC 4997. Over several years (1968-1985) it exhibited a gradual decline in the total $U B V$-brightness which, on the whole, reached $0 .^{m} 4-0 .^{m} 5$. The decline had abruptly ceased in 1985-1986, but since late 1986 to the present the nebula began to brighten quasi-linearly in all three bands. Most spectral characteristics of IC 4997 - the brightest emission lines and the continuum of the central star - were found to change in time. Over 1972-1992 an increase of excitation and ionization degrees of the nebula was detected. The most representative ratio $R$ - the ratio of the [OIII] $\lambda 4363$ $\AA$ and $\mathrm{H} \gamma$ fluxes, indicating well the excitation degree of nebular spectra, exhibited the greatest change: between 1972 and 1992 its value increased by more then a factor of 3 , and had reached a maximum $(\lg R=+0.28)$ in 1992, whereupon (1993-2005) it began to decrease. It appears that a real maximum could be observed somewhere within 1991 .

The detailed spectral analysis showed that over the period 1972-1992 the nebular integral electron density increased from $N_{e} \approx(2-6) \times 10^{5} \mathrm{~cm}^{-3}$ to $N_{e} \approx(1-3) \times 10^{6}$ $\mathrm{cm}^{-3}$, and the integral electron temperature rose from $T_{e} \approx 12000 \mathrm{~K}$ to $T_{e} \approx 14000 \mathrm{~K}$. It should be noted, that estimates of $N_{e}$ and $T_{e}$, and their temporal variations should be considered as mean, integrated estimates which characterize the object as a whole. 
Since this nebula has a complicated structure (e.g. Miranda, Torrelles \& Eiroa (1996); Miranda, Torrelles \& Eiroa (1997)), the parameters of its individual components can markedly differ from means.

The changes in continuous spectrum of IC 4997 indicate that the effective temperature of the central star and its ultraviolet flux have increased. The measuring of continuum, together with examination of earlier estimates of $T_{*}$, permits one to conclude that over the period 1972-1992 $T_{*}$ increased from $T_{*} \approx 40000 \mathrm{~K}$ to $60000 \mathrm{~K}$, or even more. Such a rise of $T_{*}$ and thus the rise of the ultraviolet flux from the central star can testify to the instability of this star; no doubt, that it had caused the revealed increase of $N_{e}$ and $T_{e}$ in the nebula. Our observations showed that intensification of the spectral activity of IC 4997, which started in the late 1960s, appears to have terminated in 1990-1992, while in 1993-2005 the activity began to decrease. The detailed results of this study, concerning the planetary IC 4997 and its variability, have been published by Kostyakova (1999) and Kostyakova (2002).

The bright $\left(\sim 7^{\prime \prime} \times 16^{\prime \prime}\right)$ planetary nebula NGC 6572 had also shown noticeable fluctuations of brightness and spectrum. Our photoelectric data revealed that over the first ten years of observations (1968-1978) the visual ( $V$ and $B$ ) brightness had undergone marked increases, which, on the whole, reached $0 .{ }^{m} 4-0 .{ }^{m} 5$. Other authors (Feibelman, Aller, \& Hyung (1992)) also noted the rapid rise of the nebula brightness over the period 1966-1973, reaching $\sim 0{ }^{m} 3-0 .^{m} 4$ in filter $V$ and $0 .{ }^{m} 2$ in $B$. Over following 25 years the visual brightness of NGC 6572 has undergone quasiperiodic (of order of two decades) fluctuations in the range of $0 .{ }^{m}$. The analysis of our spectral data, analogous to that undertaken by Kostyakova (1999), shows that over the whole period of observation the integral electron density $N_{e}$ of this nebula increased at least tenfold: in the $1960 \mathrm{~s}$ (according to various data) it was of order $n \times 10^{4} \mathrm{~cm}^{-3}$, while over 1972-2005 it rose from $n \times 10^{5} \mathrm{~cm}^{-3}$ to $n \times 10^{6} \mathrm{~cm}^{-3}$. The integral electron temperature of NGC 6572 over the whole observational period changed from $T_{e} \approx 12000-14000 \mathrm{~K}$ to $T_{e} \approx 14000-15000 \mathrm{~K}$. However, in the period of maximum activity (1987-1991) $T_{e}$ could be even higher and could reach 16000-20000 K. The examination of NGC 6572's continuum led to the conclusion that the central star became somewhat bluer; that means its ultraviolet energy flux had increased. We can assume that the effective temperature $T_{*}$ had increased from $\sim 48000 \mathrm{~K}$ to $\sim 53000 \mathrm{~K}$. (with the possibility to be higher during 1985-1992).

Finally, we can conclude that the studied variable planetaries are in an active stage of their development; therefore they can serve as tools for examing the evolution of planetary nebulae and their progenitors.

\section{References}

Arkhipova, V.P., Kostyakova, E.B., \& Noskova, R.I., 1994 Pis'ma Astron. Zh., 1994, 20, 122 Feibelman, W.A., Aller, L.H., \& Hyung, S. PASP, 1992, 104, 339

Kostyakova, E.B., Arkhipova, V.P. \& Savel'eva, M.V.,Mem. Soc. Roy. Sci. de Liege, 1973, 6 ser., V, 473

Kostyakova, E.B., Pis'ma Astron. Zh., 1990, 16, 1085

Kostyakova, E.B., Tr. Gos. Astron. Inst. Sternberg, 1991, 62, 143

Kostyakova, E.B., IAU Symp. No 180, Habing, H.J. and Lamers, H.J., Eds., Dordecht: Kluwer Acad. Publ., 1997, 251

Kostyakova, E.B. 1999, Astronomy Letters 25, 389

Kostyakova, E.B., Rev. Mex. AA (Serie de Conferencias), 2002, 12, 167

C. Miranda, L.F., Torrelles, J.M., and Eiroa, C., Astrophys. J. Lett., 1996, 461, L111

C. Miranda, L.F., Torrelles, J.M., and Eiroa, C., IAU Symp. No. 180, Habing, H.J. and Lamers, H.J., Eds., Dordecht: Kluwer Acad. Publ., 1997, 360 